\title{
Graph-Drawing Contest Report
}

\author{
Peter Eades ${ }^{1}$, Joe Marks², Petra Mutzel ${ }^{3}$, and Stephen North ${ }^{4}$ \\ 1 Department of Computer Science, University of Newcastle \\ University Drive - Callaghan, NSW 2308, Australia \\ eades@cs.newcastle.edu.au \\ 2 MERL-A Mitsubishi Electric Research Laboratory \\ 201 Broadway, Cambridge, MA 02139, U.S.A. \\ marks@merl.com \\ 3 Max-Planck-Institut für Informatik \\ Im Stadtwald, 66123 Saarbrücken, Germany \\ mutzel@mpi-sb.mpg.de \\ 4 AT\&T Research
}

180 Park Ave., Bldg. 103, Florham Park, NJ 07932-0971, U.S.A.

north@research.att.com

\begin{abstract}
This report describes the Fifth Annual Graph Drawing Contest, held in conjunction with the 1998 Graph Drawing Symposium in Montreal, Canada. The purpose of the contest is to monitor and challenge the current state of the art in graph-drawing technology [4, 5, 6, 7].
\end{abstract}

\section{Introduction}

Text descriptions of the four categories for the 1998 contest are available via the World Wide Web (WWW) [8]. Approximately 17 submissions were received, including two videos and two live demonstrations. The winners for Categories $\mathrm{A}-$ $\mathrm{C}$ were selected by a committee of experts (whose names are listed in the acknowledgements). The winner for Category D was selected by vote of all the symposium attendees. Conflicts of interest were avoided on an honor basis. The winning entries are described below.

\section{Winning Submissions}

\subsection{Category A}

The theme for Category A was incremental/dynamic graph drawing. The data consisted of addition and deletion operations that specify how a graph depicting a fragment of the WWW changes over time.

The judging committee did not award a first place in this category. However, two submissions were awarded "Honorable Mention" and split the prize fund. Although the contest rules did not specify a submission format for this category, the two top submissions were both recorded animations. 
Figure 1 shows a single frame from the submission of U. Brandes (Ulrik.Brandes@uni-konstanz.de), V. Kääb, A. Löh, D. Wagner, and T. Willhalm, University of Konstanz, Germany. They use an energy-based layout algorithm that produces a straight-line 3D drawing. It favors downward-pointing edges and penalizes excessive movement between consecutive layouts. In addition, the viewpoint is updated automatically to keep the whole graph in view, to minimize the change in viewer position, and to avoid occlusions. The actual Web pages are texture-mapped onto the sides of the cubic nodes, and can be read when the viewer zooms in.

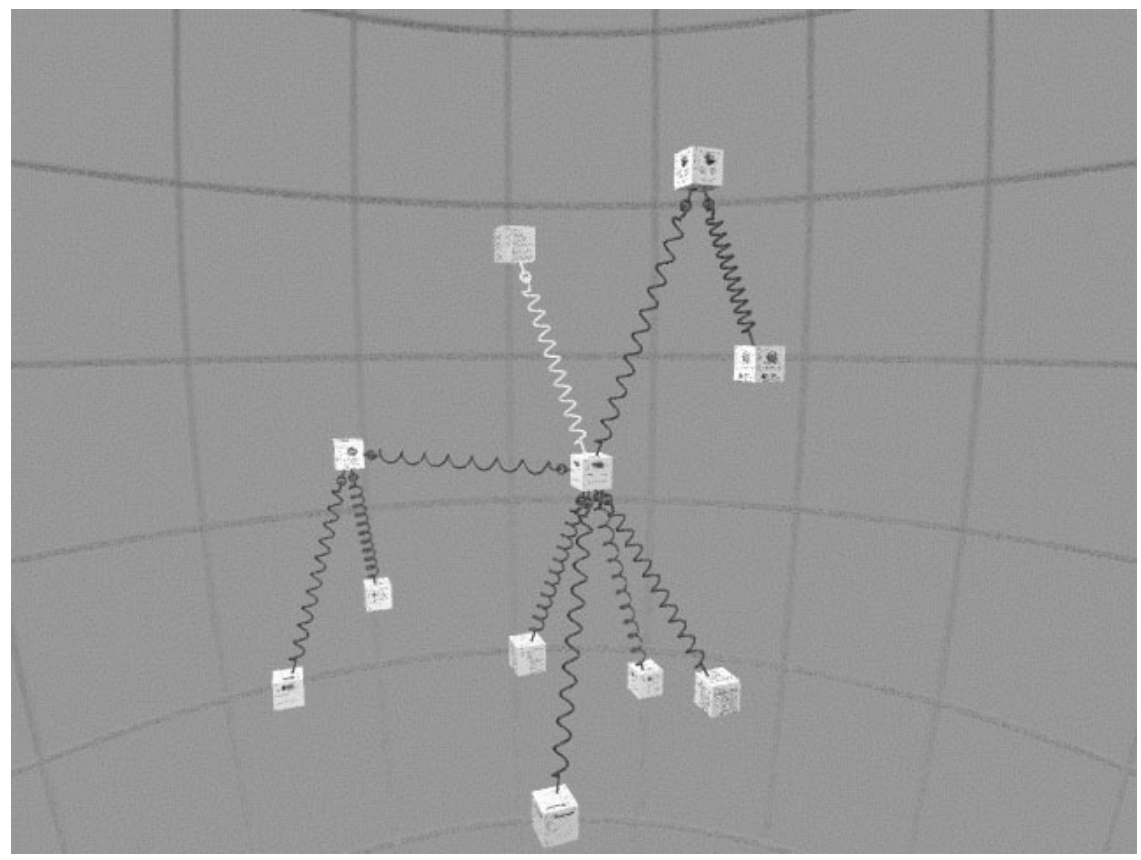

Fig. 1. Honorable mention, Category A (original in color).

The final frame from the submission of Thomas Wurst, (wurst@informatik.uni-tuebingen.de), University of Tübingen, Germany, is shown in Figure 2, The Inca incremental graph-drawing algorithm was developed within the GRAVIS system [9]. It is similar to the well-known Sugiyama layout algorithm, except that the assignment of nodes to layers is not done to minimize edge crossings, but instead takes into account the relative positions of the nodes already there in an attempt to preserve the user's "mental map" of the drawing. Note also the use of diagonally oriented text labels, a simple technique originally used by Schreiber and Friedrich [5]. 


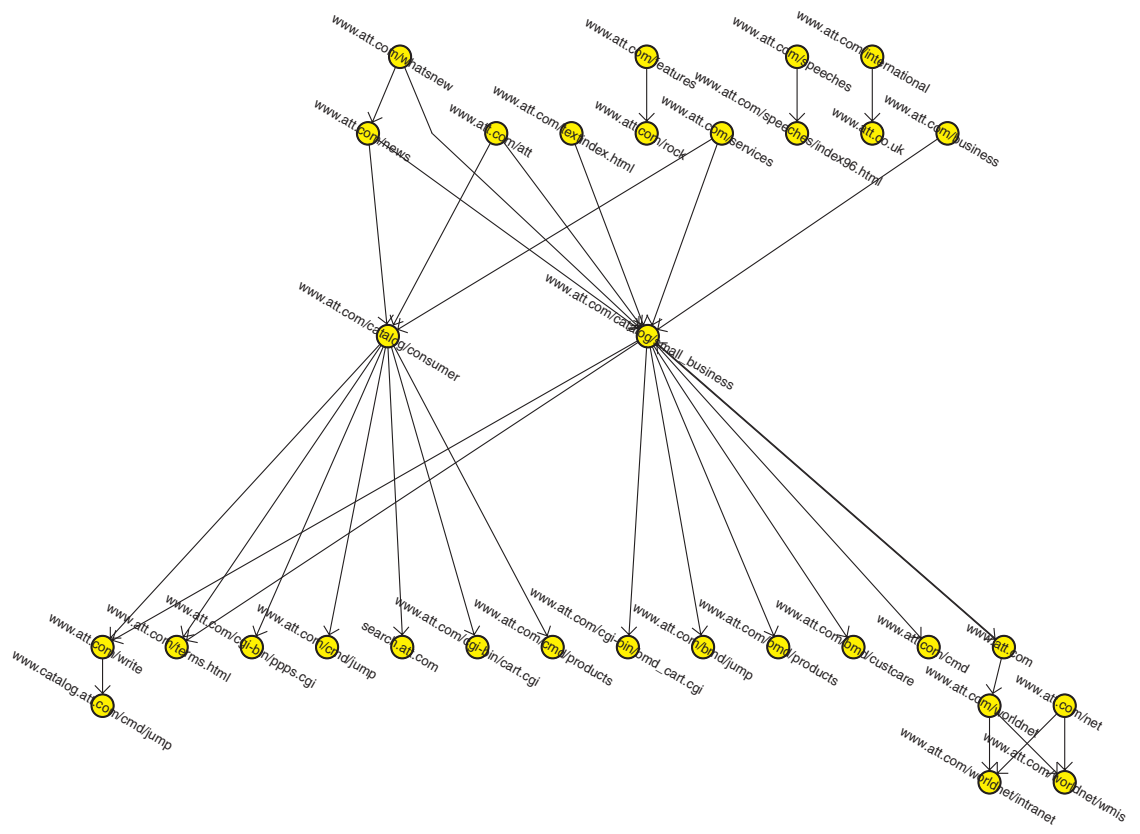

Fig. 2. Honorable mention, Category A (original in color).

\subsection{Category B}

The graph for this category was provided by Siemens AG, and is typical of graphs that arise in the context of computer-integrated manufacturing. The vertices represent various states of a manufacturing machine, and the relations between them represent the possible state transitions. Each state transition is modeled as a path of length two with a vertex (smaller than the main vertices) in between. There are also text labels or tags assigned to some of the vertices (main or subvertices) 11 The original hand drawing looked very confusing; approximately two weeks of laborious manual editing were needed to refine it. The refined hand drawing is shown in Figure 3 .

The winning drawing is shown in Figure 4 It was produced by Vladimir Batagelj and Andrej Mrvar ([Vladimir.Batagelj, Andrej.Mrvar]@uni-lj.si) from the University of Ljubljana, Slovenia, using the "Pajek" system [10. Because symmetries in the graph (with some exceptions) were very noticeable, they decided to obtain an initial layout using a Fruchterman-Reingold spring embedder. Then they used manual editing (which took three hours) to maximize symmetries and made some adjustments to take into account the different sizes and shapes of nodes.

\footnotetext{
${ }^{1}$ Meaningless labels were substituted for the actual text to preserve confidentiality.
} 


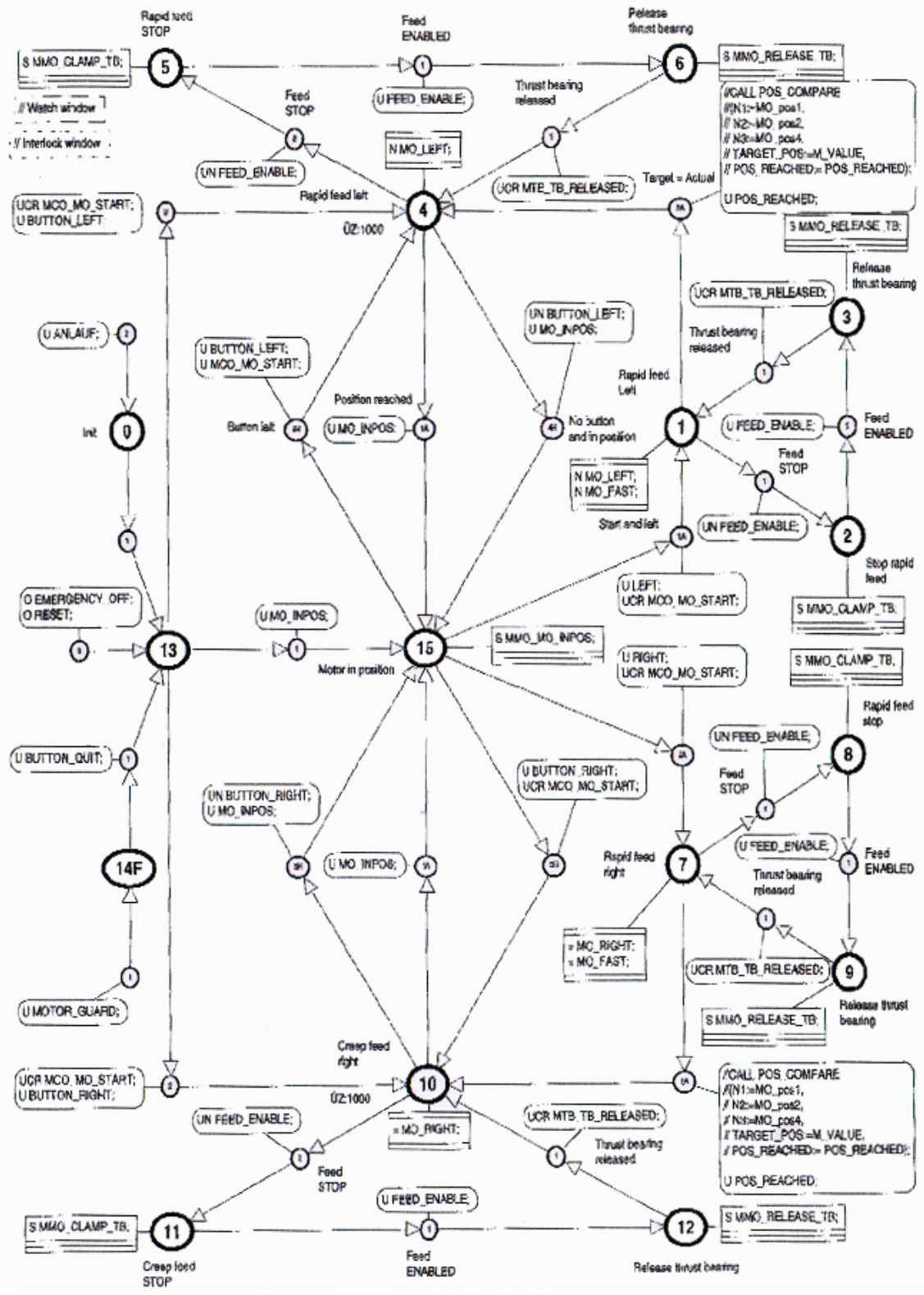

Fig. 3. Manual drawing of subject graph, Category B. 


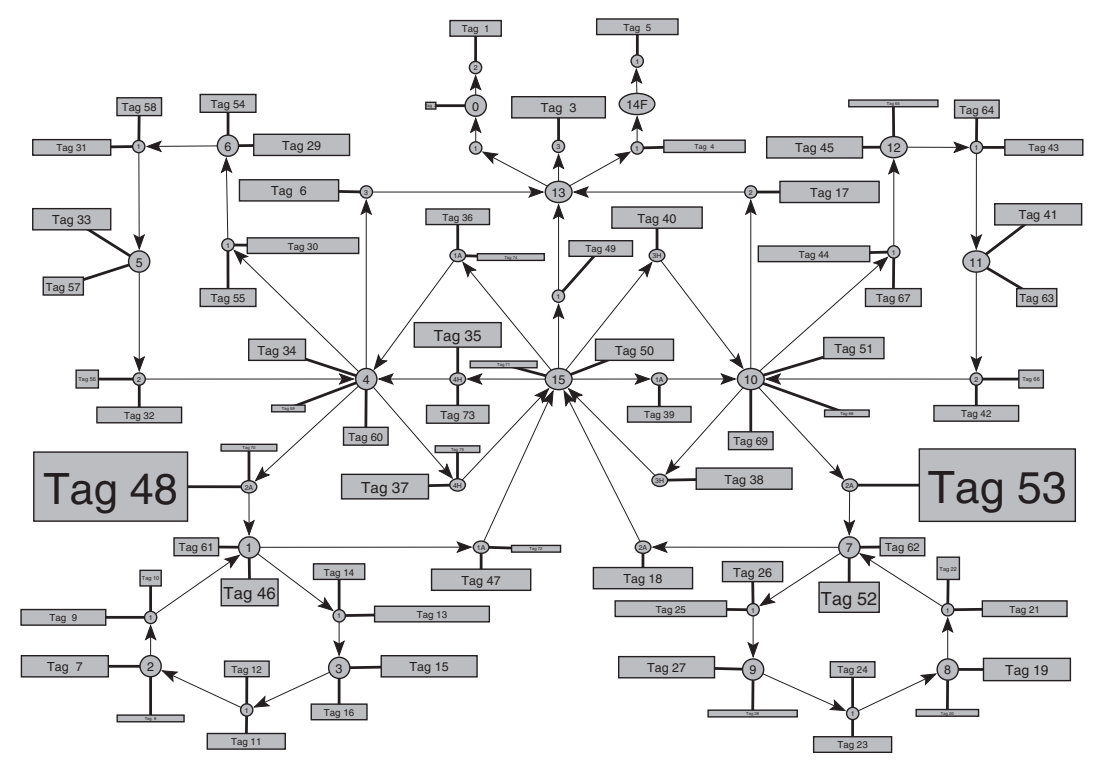

Fig. 4. First prize, Category B.

The second-place drawing for Category B is shown in Figure 5. It was submitted by Michael Wissen (wissen@mpi-sb.mpg.de) from the Max-Planck-Institute for Computer Science, Saarbrücken, Germany. He used a new graph-drawing algorithm that is particularly suited to small graphs. The vertex-placement step of the algorithm is based on so-called region trees and aims at placing the vertices so that they split some region (see [12]). The labelling step uses a simple greedy algorithm. The drawing was generated fully automatically.

\subsection{Category $\mathrm{C}$}

The only information given out for Category $\mathrm{C}$ was that it involved an elegant graph of theoretical significance. In fact, the graph has girth 11 (i.e., no cycles of length less than 11), degree 3, and 112 vertices. A graph with a minimum number of vertices for a given degree and given girth is a cage. It has been shown recently that there are no graphs of girth 11 and degree 3 with fewer vertices. A manual drawing [1, 2] is shown in Figure 6]

Several researchers reported that they found it very difficult to make sense of this graph using standard graph-layout algorithms. The top two drawings both took novel approaches to the analysis and subsequent layout of the graph. First prize was awarded to Petrus Abri Santoso (Peasant@acm.org) and Andi 


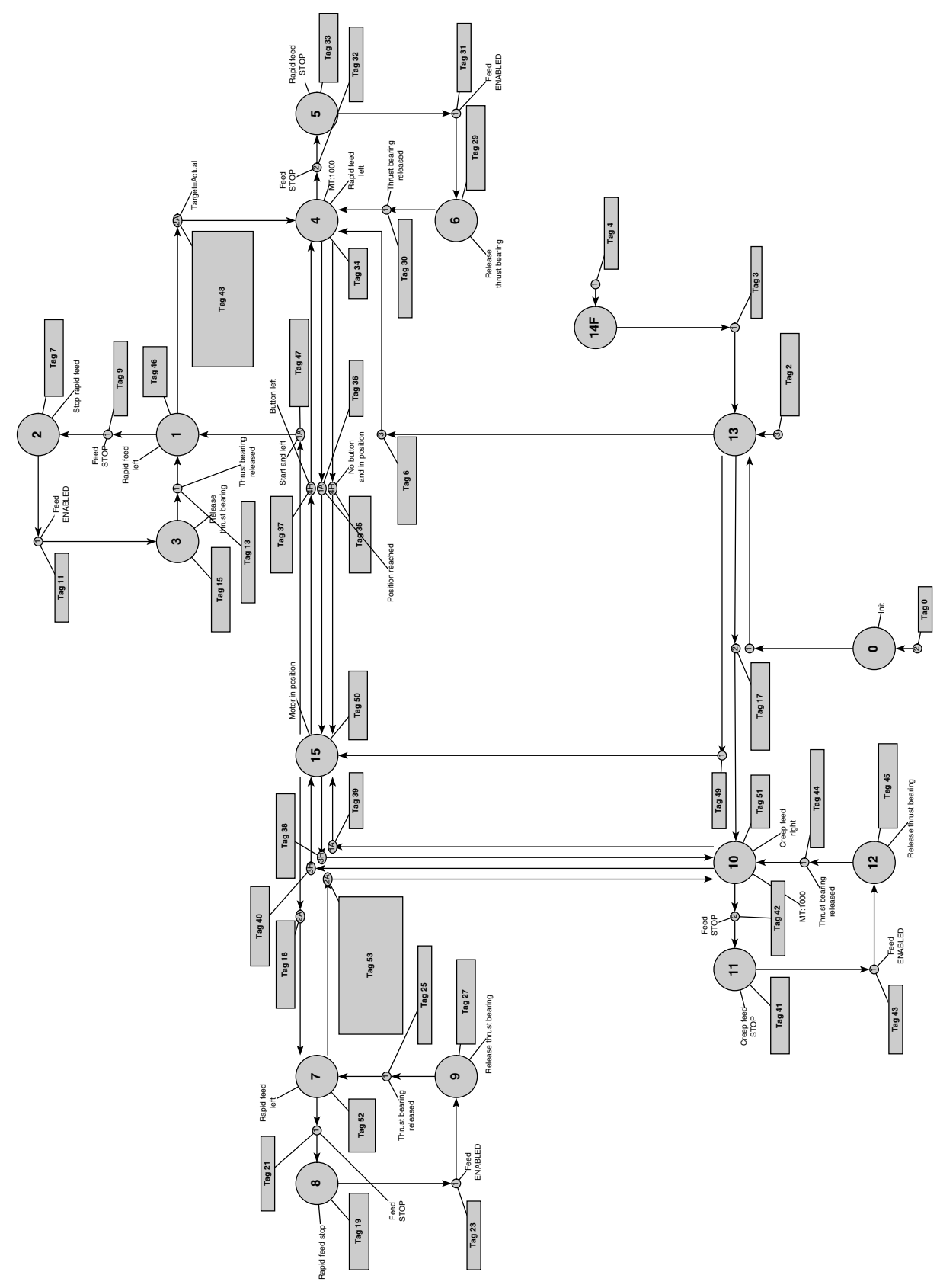

Fig. 5. Second prize, Category B. 


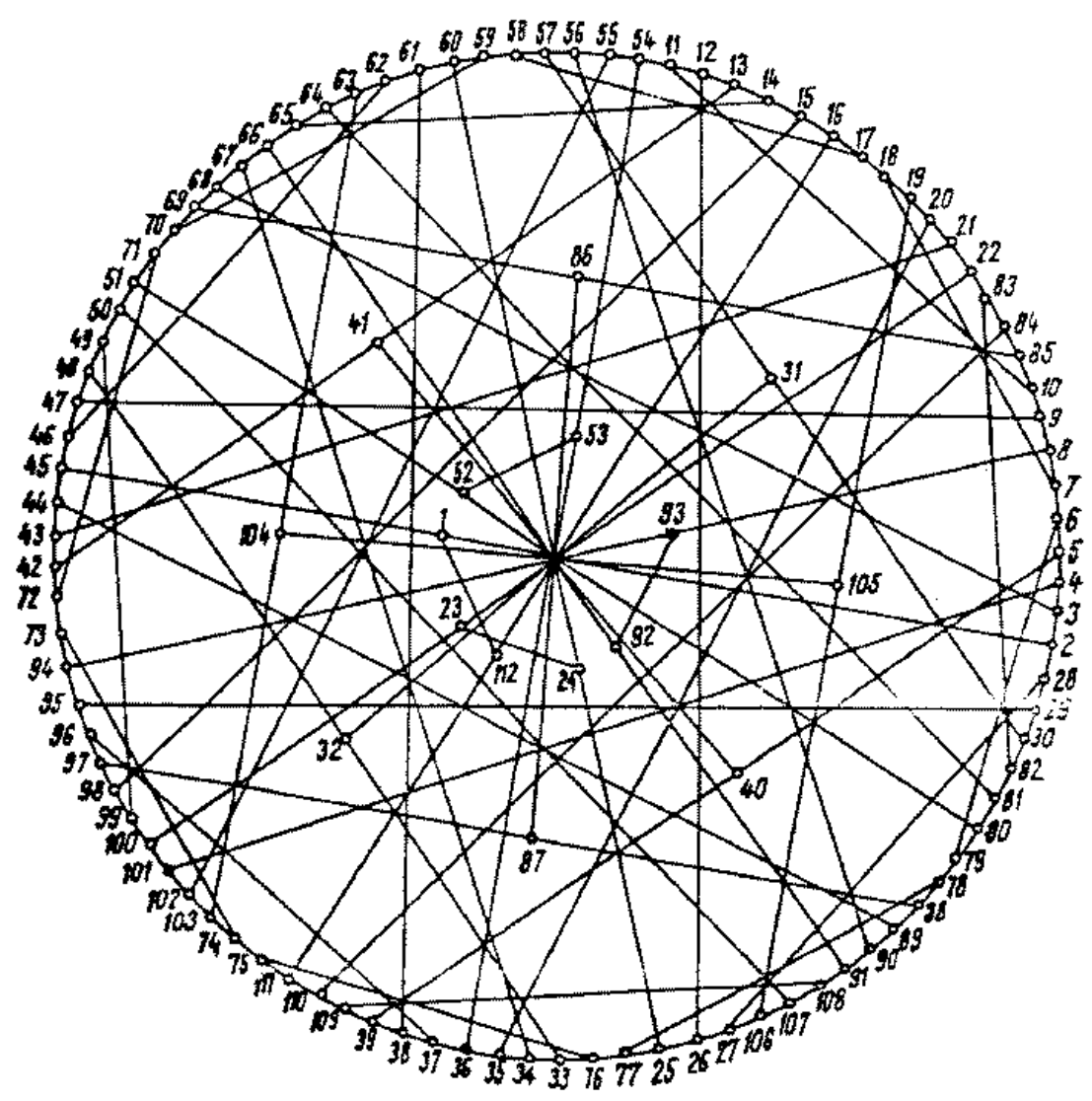

Fig. 6. Manual drawing of subject graph, Category C.

Surjanto (Asurjant@gamma.binus.ac.id) from the Bina Nusantara University, Jakarta, Indonesia, for the drawing in Figure 7 . They began by dividing the graph nodes according to their eccentricity. Nodes of common eccentricity were positioned on their own circle. The arrangement and coloring of nodes in each circle were then refined to emphasize the symmetry in the graph.

Second prize was awarded to Egon Pasztor (pasztor@merl.com), from MERLA Mitsubishi Electric Research Laboratory, Cambridge, Massachusetts, U.S.A., and Doris Tsao (dtsao@fas.harvard.edu), from Harvard University, Cambridge, Massachusetts, U.S.A. They began with an analysis of the sums of successive powers of the graph's adjacency matrix, which revealed eight exceptional pairs of nodes that were separated by a longer shortest path than all others. By manually separating these nodes and gathering their neighbors, the graph was seen to be two sets of eight, trilevel binary trees. The leaves of these trees are connected by 64 edges, which represent an awkward permutation. Drawing the graph well 


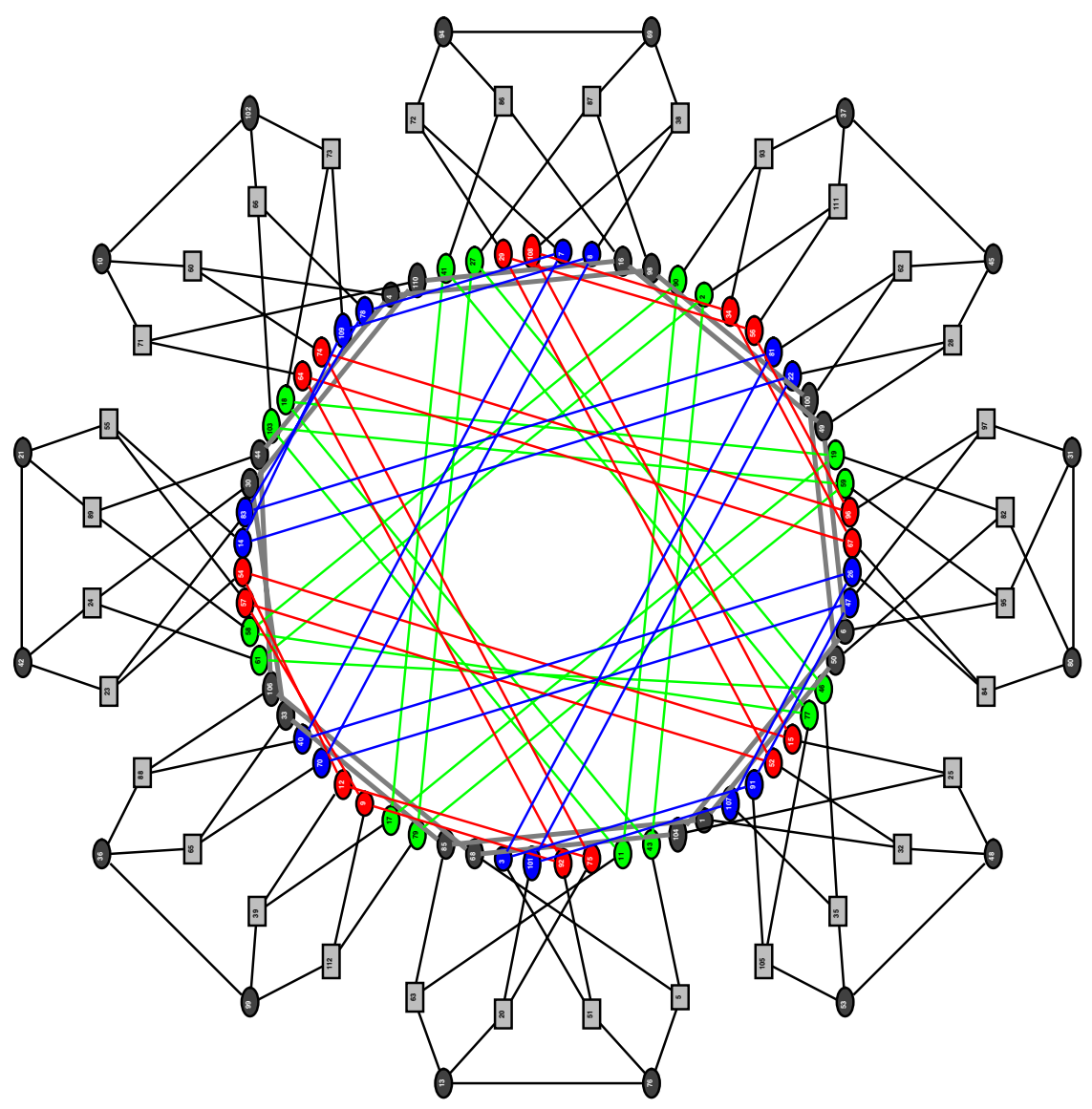

Fig. 7. First prize, Category C (original in color).

therefore seemed to reduce to the problem of drawing the permutation in an orderly way. This was accomplished using a custom program that allows the user to interactively reorganize the crossing of a permutation. A more complete description of the permutation-crossing program and this unusual drawing is available on the WWW 11].

\subsection{Category D}

The only requirement for submissions in this category was that they be some form of artistic expression inspired by or related to graph drawing.

The clear winner in this category was submitted by Roland Wiese (wiese@informatik.uni-tuebingen.de) from the University of Tübingen, Germany. The drawing in Figure 9 contains four copies of $K_{50}$. The nodes are arranged so that they form a big blue circle in the center of the drawing. An orthogonal drawing 


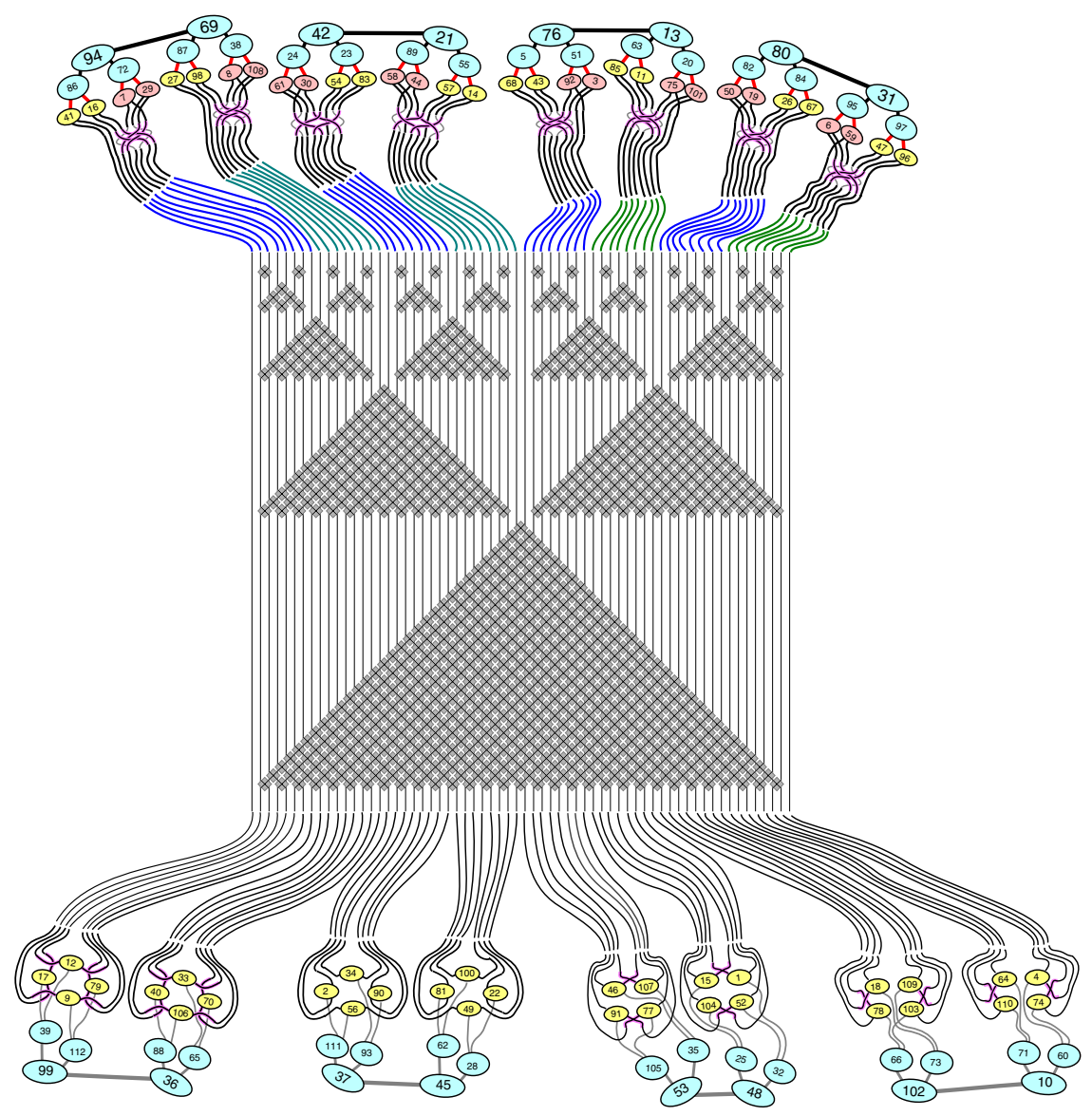

Fig. 8. Second prize, Category C (original in color).

algorithm was used to compute the layout of the $K_{50}$ graph. It was implemented in the GraVis system [9].

Three other submissions were awarded joint second prize. The ASCII drawings in Figure 10 were created by Therese Biedl (therese@cs.mcgill.ca), who devised a family of ASCII-character patterns for lines of different slopes, using only symbols for which the symmetric symbol (with respect to the y-axis) also exists.

The two other submissions to share second prize were both videos. Figure 11 shows 10 keyframes taken from an animation in which multiple drawings of $K_{3,5}$ blend one into the next. The set of drawings was generated automatically to balance diversity and aesthetics [3]. This was joint work by: Therese Biedl, McGill University, Canada; Joe Marks, MERL-A Mitsubishi Electric Research Labo- 
ratory, U.S.A. (marks@merl.com); Kathy Ryall, University of Virginia, U.S.A.; and Sue Whitesides, McGill University, Canada.
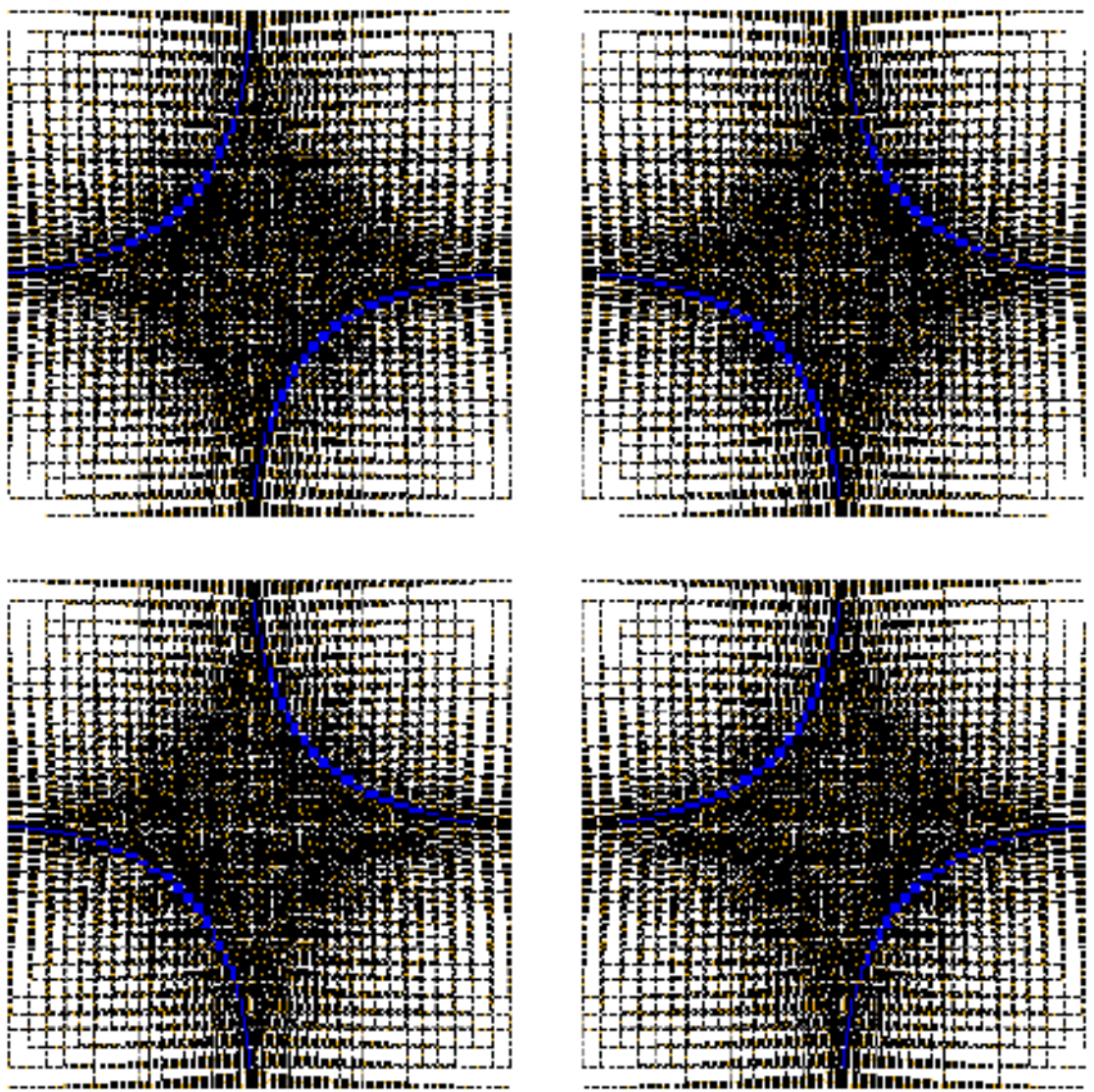

Fig. 9. First prize, Category D (original in color).

Figure 12 shows a still from a video depicting a WWW site using an interactive 3D graph-drawing system called IN3DNET. It was submitted by Marco Sbarrini (sbarrini@datamat.it) and Valerio Violi from the Third University of Rome, Italy.

\section{Observations and Conclusions}

The exceptionally high quality and originality of this year's contest submissions demonstrates how far the field has progressed in the last few years. 

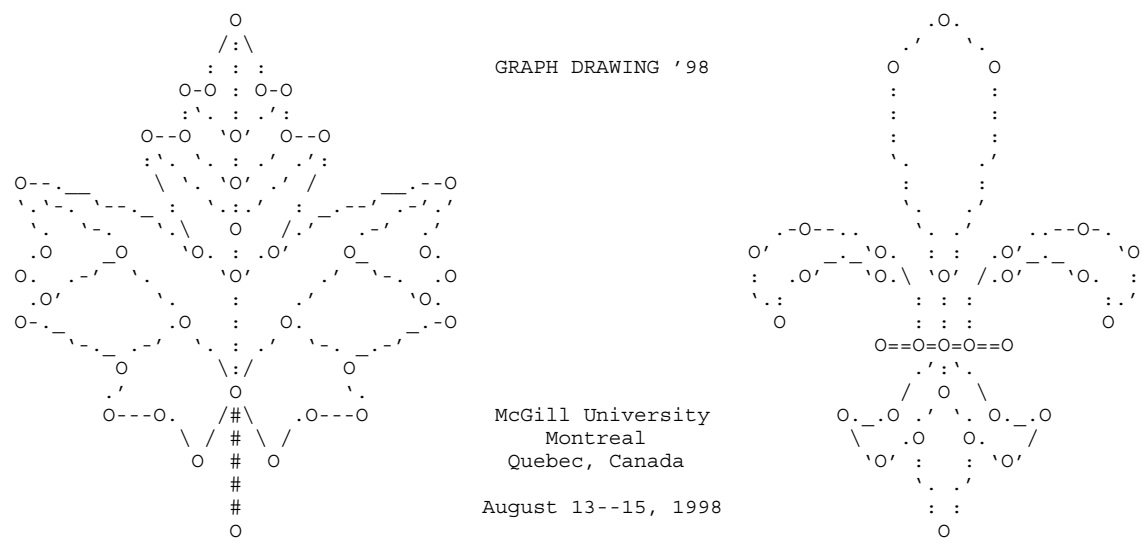

Fig. 10. Joint second prize, Category D.

Incremental layout still remains a challenge for the Graph Drawing community, and no doubt will continue to be included in future contests. However, the submissions for Category A showed that work on incremental layout and diagram animation is providing approaches that are at least partially successful at both preserving a user's mental map and at manifesting graph-structure changes. Video submissions clearly had an advantage in demonstrating incremental-layout capabilities. The continued encouragement of video submissions should also help to stimulate applications of $3 \mathrm{D}$ layout.

The entries for Categories B and C suggest worthwhile directions for future graph-drawing research (such as identifying and exploiting internal graph structure, handling variable-sized nodes well, and supporting text annotation). As in past years, most of the winners combined automated and manual techniques to great effect. It is worth noting that the two winners in Category $\mathrm{C}$ both used graph-analysis techniques (i.e., measures of eccentricity, etc.) that are not computed by many commercial and academic graph-drawing systems.

Category D, the "artistic" category, was received with much enthusiasm, and will be continued next year.

The variety of approaches represented by the contest winners, and the enthusiasm for the competition is encouraging, and we want to take this opportunity to thank all the entrants again.

\section{Acknowledgements}

Sponsorship for this contest was provided by Siemens AG, AT\&T Research, MERL-A Mitsubishi Electric Research Laboratory, and Tom Sawyer Software. 

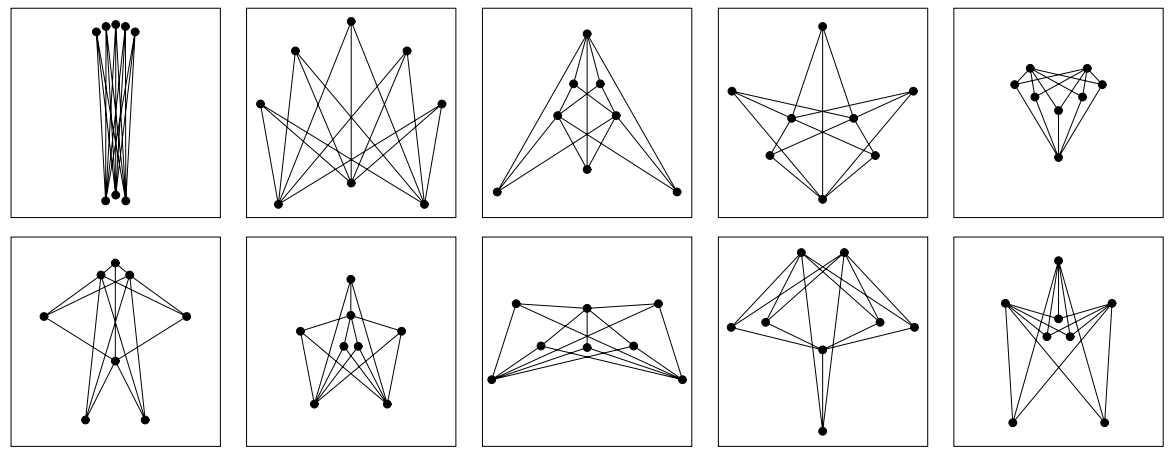

Fig. 11. Joint second prize, Category D.

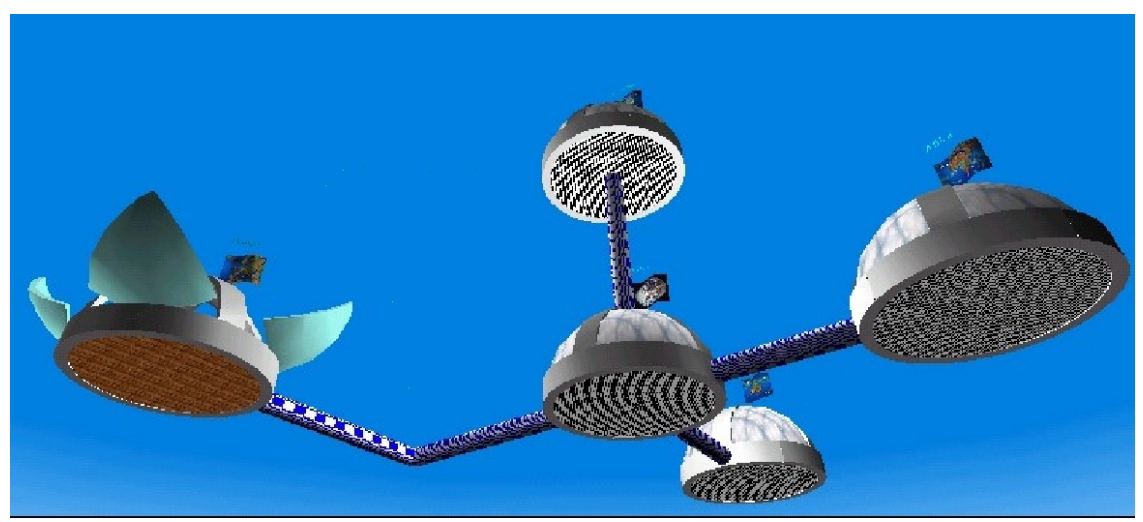

Fig. 12. Joint second prize, Category D (original in color).

Robin Chen, Henry Kulzer, Brendan McKay, and Gordon Royle contributed graph data for the contest. Peter Eades, Michael Jünger, Joe Marks, Petra Mutzel, and Stephen North served on the judging committee. Jan Kratochvil acted as arbiter and auditor.

\section{References}

[1] A. T. Balaban. Trivalent graphs of girth nine and eleven and relationships between cages. Rev. Roum. Math. Pures Appl., 18:1033-1043, 1973.

[2] A. T. Balaban. Solved and unsolved problems in chemical graph theory. In J. Gimbel, J. Kennedy, and L. Quintas, editors, Quo Vadis, Graph Theory? (Annals of Discrete Mathematics, Vol. 55), pages 109-126. 1993.

[3] T. Biedl, J. Marks, K. Ryall, and S. Whitesides. Graph multidrawing: Finding nice drawings without defining nice. In this volume. 
[4] P. Eades and J. Marks. Graph-drawing contest report. In R. Tamassia and I. G. Tollis, editors, Lecture Notes in Computer Science: 894 (Proceedings of the DIMACS International Workshop on Graph Drawing '94), pages 143-146, Berlin, October 1994. Springer.

[5] P. Eades and J. Marks. Graph-drawing contest report. In F. J. Brandenburg, editor, Lecture Notes in Computer Science: 1027 (Proceedings of the Symposium on Graph Drawing GD '95), pages 224-233, Berlin, September 1995. Springer.

[6] P. Eades, J. Marks, and S. North. Graph-drawing contest report. In S. North, editor, Lecture Notes in Computer Science: 1190 (Proceedings of the Symposium on Graph Drawing GD '96), pages 129-138, Berlin, September 1996. Springer.

[7] P. Eades, J. Marks, and S. North. Graph-drawing contest report. In G. DiBattista, editor, Lecture Notes in Computer Science: 1353 (Proceedings of the Symposium on Graph Drawing GD '97), pages 438-445, Berlin, September 1997. Springer.

[8] http://gd98.cs.mcgill.ca/contest/.

[9] http://www-pr.informatik.uni-tuebingen.de/Gravis/Gravis.html.

[10] http://vlado.fmf.uni-lj.si/pub/networks/pajek/.

[11] http://www.merl.com/people/pasztor/graphContest.

[12] M. Wissen. Automatisches Zeichnen von Zustandsdiagrammen. Master's thesis, Diplomarbeit, Fachbereich Informatik, Universität des Saarlandes, 1998. 